\title{
Efecto de ocho portainjertos sobre la producción de dos variedades de cerezo
}

Tersoglio, E. y Setien, N.

DOI 10.31047/1668.298x.v38.n2.27429

\begin{abstract}
RESUMEN
Los portainjertos mejoran la adaptación de la variedad a diferentes condiciones ambientales e incrementan la producción. Se midió la influencia de los portainjertos SL 64, MxM 60, MxM 14, CAB 6P, Weiroot 13, GiSeLa® 5, GiSeLa® 6 y Mariana 2624 con filtro Adara, sobre la producción de dos variedades de cerezo. El diseño experimental fue por bloques completos al azar, con seis repeticiones. Las variables fueron: producción cosechada $\left(P_{\mathrm{rFC}}\right)$, afinidad productiva $\left(A_{f}\right)$, precocidad del árbol $\left(P_{\text {rec }}\right)$, producción fruta sana $\left(P_{r F S}\right)$, fracción de pérdida $\left(f_{P e r}\right)$ y volumen de dosel $\left(V D_{\mathrm{rt}}\right)$. Salinidad edáfica asoció con mayor $\mathrm{f}_{\mathrm{Per}}$ y menor $\mathrm{P}_{\mathrm{rFC}}, \mathrm{P}_{\mathrm{rFS}}$ y $\mathrm{P}_{\mathrm{rec}}$. Variedad influyó sobre $\mathrm{P}_{\mathrm{rFS}}$ y $\mathrm{f}_{\mathrm{Per}}$; portainjerto lo hizo sobre $P_{r E C}$ y $P_{r E S}$ e interacción lo hizo sobre $P_{r E C}, P_{r F S}$ y $f_{P e r}$. $V D_{c t}$ fue un predictor impreciso de $P_{r F C}$ y de $P_{r F S}$ pero, $P_{r F C}$ fue un predictor robusto de $P_{\text {rFs. }}$. En las condiciones del ensayo, Bing sobre MxM 60 o Weiroot 13 y Celeste sobre SL 64, MxM 60 o Adara-M2624 tuvieron alta $P_{\mathrm{rFS}}$, buena adaptación edafoclimática, precocidad y la mayor $P_{\mathrm{rFS}}$ en condiciones de lluvia durante la cosecha. Las combinaciones de Bing sobre MxM14, GiSeLaß 6 o Adara-M2624 registraron alta $P_{\mathrm{rFS}}$ pero con diferencias de adaptación edafoclimática. La afinidad variedad-portainjerto fue altamente específica.
\end{abstract}

Palabras clave: producción de fruta sana, fracción de pérdida, precocidad, afinidad productiva

Tersoglio, E. y Setien, N., 2021. Effect of eight rootstocks on the production of two sweet cherry varieties. Agriscientia 38 (2): 63-73

\section{SUMMARY}

Rootstocks improve variety adaptation to different environmental conditions and increases production. The influence of SL 64, MxM 60, MxM 14, CAB 6P, Weiroot 13, GiSeLa ${ }^{\circledR}$ 5,GiSeLa ${ }^{\circledR} 6$ and Mariana 2624 with Adara filter as rootstocks was measured on the production of two sweet cherry varieties. The experimental design was a randomized complete block design with six replications. The variables were: harvested fruit production per tree $\left(\mathrm{P}_{\mathrm{rFC}}\right)$, 
production affinity $\left(A_{f}\right)$, tree precocity $\left(P_{\text {rec }}\right)$, damage-free fruit production $\left(P_{r F S}\right)$, rejected fruit fraction $\left(f_{P e r}\right)$ and canopy volume $\left(V D_{c t}\right)$. Edaphic salinity correlated with higher $f_{\mathrm{Per}}$ and lower $\mathrm{P}_{\mathrm{rFC}}, \mathrm{P}_{\mathrm{rFS}}$ and $\mathrm{P}_{\text {rec }}$. Variety influenced $\mathrm{P}_{\mathrm{rFS}}$ and $f_{P e r}$; rootstock influenced $P_{r F C}$ and $P_{r F S}$, and interaction influenced $P_{r F C}, P_{r F S}$ and $f_{\mathrm{Per}} \cdot V D_{\mathrm{Ct}}$ was an imprecise predictor of $P_{\mathrm{rFC}}$ and $P_{\mathrm{rFS}}$ but, $P_{\mathrm{rFC}}$ was a robust predictor of $P_{r F S}$. Under experimental conditions, Bing on MxM 60 or W 13 and Celeste on SL 64, MxM 60 or Adara-M2624 had high $P_{r F S}$, good soil-climate adaptation, precocity and the highest $\mathrm{P}_{\mathrm{rFS}}$ in rainy conditions at harvest. The combinations of Bing on MxM 14, GiSeLa® 6 or Adara-M2624 showed high $\mathrm{P}_{\mathrm{rFS}}$, but differences in soil-climate adaptation were observed. The variety-rootstock affinity was highly specific.

Keywords: damage-free fruit production, rejected fruit fraction, precocity, production affinity

Tersoglio, E. (ORCID: 0000-0002-0869-4858) y Setién, N. (ORCID: 0000-0002-5870-3601): Instituto Nacional de Tecnología Agropecuaria (INTA), Estación Experimental Agropecuaria Mendoza., Av. San Martín 3853, Luján de Cuyo, Mendoza, Argentina. Correspondencia a: tersoglio.eduardo@inta.gob.ar

\section{INTRODUCCIÓN}

Los portainjertos son un componente esencial de la fruticultura moderna debido a su capacidad de adaptar las variedades a diferentes condiciones ambientales. Estos proporcionan cualidades que están ausentes en la variedad, como resistencia a plagas y enfermedades de suelo, mejor anclaje, absorción de nutrientes y tolerancia a la hipoxia, entre otros. Además, interaccionan con la variedad, dando características productivas específicas ya que modifican el vigor que permiten huertos de diferentes densidades de plantación (Aglar y Yıldız, 2014; Fajt, Jug et al., 2014; Sansavini y Lugli, 2014; Gainza et al., 2015). También influyen en el rendimiento, la precocidad y la arquitectura de la planta (Whiting et al., 2005) y la eficiencia productiva y la de cosecha (Ampatzidis y Whiting, 2013).

Los antecedentes indican que los portainjertos afectan la producción por árbol, la eficiencia de rendimiento (Fajt, Folini et al., 2014; LópezOrtega et al., 2016) y los atributos de calidad de la fruta (Gonçalves et al., 2006). Además, un mismo portainjerto tiene diferentes desempeños según las condiciones agroclimáticas de la región, destacando la importancia de la selección local de los materiales genéticos mejor adaptados (Gonçalves et al., 2006; Usenik et al., 2006; Sansavini y Lugli, 2014; Ljubojevic et al., 2016) y en especial, los portainjertos restrictivos (Fajt,
Folini et al., 2014). En el mundo, la causa de pérdida de mayor importancia, por su intensidad y frecuencia, es el agrietado. Este proceso se asocia a la absorción directa y pasiva de agua de lluvia a través de la membrana cuticular que es hidrófoba, pero discontinua. Tales fracturas permiten la entrada a través de la epidermis del fruto (Winkler et al., 2016). Además, existe una vía indirecta, que es la absorción de agua a través de las raíces (Measham et al., 2010; Measham et al., 2014). Ambos caminos serían complementarios y provocan una expansión de las paredes celulares de la epidermis, una ruptura de la membrana cuticular luego, una falla de la epidermis e hipodermis con la consecuente formación de grietas visibles que liberan la tensión (Schumann y Knoche, 2020). En tal sentido, la combinación variedad-portainjerto ideal es aquella que induce baja proporción de pérdida en temporadas con condiciones climáticas adversas.

Es muy importante para la industria local la selección de portainjertos con buena adaptación edafoclimática que permitan alcanzar temprana y frecuentemente alta producción de calidad, mejorando la sostenibilidad económica (Whiting et al., 2005; Seavert y Long, 2007; López-Ortega et al., 2016) y que reduzcan la huella de carbono (Rana et al., 2019).

Los portainjertos de cerezos utilizados con mayor frecuencia en la provincia de Mendoza son: Prunus avium L., Prunus mahaleb L. y más recientemente 
la selección CAB 6P de Prunus cerasus L. Aunque, con desempeños aceptables, cada uno presenta ventajas y limitaciones. Actualmente, $P$. avium es poco utilizado, por su elevado vigor y baja precocidad. P. mahaleb clon SL 64 tiene elevada susceptibilidad a la hipoxia, aunque es resistente a sequía (Jiménez et al., 2007; López-Ortega et al., 2016). El portainjerto CAB 6P es de vigor intermedio y productivo (Sansavini y Lugli, 2014), tiene buena sobrevivencia (Pimentel et al., 2014) pero baja precocidad (Gratacós et al. 2007) y es sensible a la salinidad (Papadakis et al., 2018).

El objetivo del trabajo fue establecer la influencia de ocho portainjertos sobre la producción de fruta cosechada y la de fruta sana, libre de daños y defectos, de dos variedades de cerezos, en las condiciones agroclimáticas locales.

\section{MATERIALES Y MÉTODOS}

\section{Condiciones del ensayo}

El ensayo se realizó en la Estación Experimental

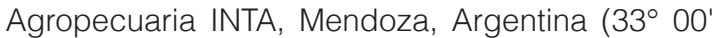
13" S, 68 51' 34" O y 920 m s.n.m.) cuyo clima es continental árido y desértico, con una temperatura media anual de $17^{\circ} \mathrm{C}$ con gran amplitud térmica. Los veranos son cálidos, con una precipitación media de $190 \mathrm{~mm}$. La oferta media de frío invernal es de 1106 unidades de frío Utah (Tersoglio y Naranjo, 2007). La profundidad del suelo oscila entre $0,90 \mathrm{~m}$ y $1,50 \mathrm{~m}$, textura franco-arcillosa con capas lenticulares horizontales compactadas.

Las características edáficas de cada bloque fueron: bloque $\mathrm{I}(\mathrm{BI}) \mathrm{con} \mathrm{CE}_{\mathrm{es}} 2,88 \mathrm{dS} \cdot \mathrm{m}^{-2}$, porcentaje de limo-arcilla (\% $\%+A) 50 \mathrm{~g} \% \mathrm{~g}$ y contenido total de $\mathrm{N}\left(\mathrm{N}_{\mathrm{T}}\right) 606$ mg.kg-1; bloque II (BII) con $\mathrm{CE}_{\mathrm{es}} 1,71$ dS.m ${ }^{-2}, \% \mathrm{~L}+\mathrm{A} 55 \mathrm{~g} \% \mathrm{~g}$ y $\mathrm{N}_{\mathrm{T}} 603 \mathrm{mg}^{\mathrm{k}} \mathrm{kg}^{-1}$; bloque III (BIII) con $\mathrm{CE}_{\mathrm{es}} 1,55 \mathrm{dS} \cdot \mathrm{m}^{-2}, \% \mathrm{~L}+\mathrm{A} 58,5 \mathrm{~g} \% \mathrm{~g}$ y $\mathrm{N}_{\mathrm{T}}$ $628 \mathrm{mg} \cdot \mathrm{kg}^{-1}$; bloque IV (BIV) con $\mathrm{CE}_{\mathrm{es}} 1,55 \mathrm{dS} \cdot \mathrm{m}^{-2}$,

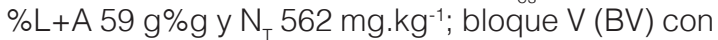
$\mathrm{CE}_{\mathrm{es}} 1,43 \mathrm{dS} \cdot \mathrm{m}^{-2}, \% \mathrm{~L}+\mathrm{A} 57 \mathrm{~g} \% \mathrm{~g}$ y $\mathrm{N}_{\mathrm{T}} 579 \mathrm{mg} \mathrm{kg}^{-1}$; bloque $\mathrm{VI}(\mathrm{BVI})$ con $\mathrm{CE}_{\mathrm{es}} 1,51 \mathrm{dS} \cdot \mathrm{m}^{-2}, \% \mathrm{~L}+\mathrm{A} 50$ $\mathrm{g} \% \mathrm{~g}$ y $\mathrm{N}_{\mathrm{T}} 627 \mathrm{mg}^{\mathrm{kg}}{ }^{-1}$.

El ensayo se inició en el 2004, con un marco de plantación de $3 \mathrm{~m}$ entre plantas y $5 \mathrm{~m}$ entre hileras, bajo malla antigranizo de $5 \mathrm{~m}$ de altura. El sistema de conducción fue de tres ejes por árbol con 6 a 7 laterales en cada uno. El riego, de cuatro líneas de goteo por hilera, aplicó una lámina horaria de 1,9 $\mathrm{mm}$ con una reposición fija de 7,6 mm cada vez que el suelo alcanzó un potencial matriz de 30-40 $\mathrm{kPa}$. Las fertilizaciones fueron de postcosecha y otoñales según Silva y Rodríguez (1995).

\section{Materiales vegetales}

Se estudió el comportamiento productivo de ocho portainjertos durante el período comprendido entre el cuarto y el octavo año después de la injertación (ADI). Los portainjertos fueron: P. mahaleb L. selección SL 64, los híbridos inter-específicos ( $P$. mahaleb x $P$. avium) selecciones MxM 60 y MxM 14, las selecciones de $P$. cerasus L. CAB 6P y Weiroot 13 (W 13), los híbridos interespecíficos ( $P$. cerasus $\times$ P. canescens) selecciones GiSeLa ${ }^{\circledR} 5$ (G 5) y GiSeLa® 6 (G 6). También se utilizó una combinación portainjerto-filtro formada por Mariana 2624 (M2624) (P. cerasifera Ehrh. x P. munsoniana W. Wight \& Hedrick) y por la selección Adara de $P$. cerasifera var. Mirobalán, respectivamente. El filtro Adara es intercompatible con M2624 y con P. avium formando un "bionte" denominado Adara-M2624 (Moreno et al., 1995). Según Tersoglio y Setien (2016) el dosel de los portainjertos SL 64 y MxM 60 se clasificó como vigoroso; el de MxM 14, CAB $6 P$ y Adara-M2624 como semivigoroso; el de W 13 como semienanizante; el de $G$ 6, enanizante y el de $\mathrm{G} 5$, muy enanizante.

Las variedades utilizadas fueron: Bing obtenida por Seth Lewelling en 1875 por semilla de la variedad Republican (Serradilla et al., 2015), clon OB260 seleccionado por la Universidad de Oregon (EE UU), vigor medio-alto, auto-incompatible grupo III, maduración, productividad y tamaño medio, excelente calidad gustativa, susceptible al agrietado; y Celeste, de vigor medio, auto-fértil grupo IX, floración media (1-2 días después de Bing) maduración temprana, productividad media alta, tendencia a la sobrecarga, pedúnculo corto, buena firmeza, muy susceptible al agrietado.

\section{Cosecha, muestreo y clasificación de frutos}

La cosecha se inició cuando, aproximadamente, un tercio de la producción alcanzó grado de color 3 en la tabla de colores de cosecha de cereza de la EEA INTA Mendoza, equivalente al código 4 de la tabla de colores Ctifl (Francia, 2005). La cosecha se hizo en una o dos pasadas dependiendo de la concentración de la madurez, pero sólo se evaluó la primera. El muestreo de cosecha fue sistemático y correspondió al $10 \%$ de la producción de las plantas que formaron cada unidad experimental (UE). La muestra se identificó y se envió al laboratorio para su análisis. En laboratorio, de cada muestra se extrajo al azar una sub-muestra, que osciló entre $1,0 \mathrm{~kg}$ y $3,0 \mathrm{~kg}$, según producción. Los frutos de cada sub-muestra se clasificaron según presencia/ausencia de daños y/o defectos. 


\section{Variables de estudio}

Producción cosechada por árbol $\left(\mathrm{P}_{\mathrm{rFC}}\right)$ es la producción media de las plantas de una UE y representa el potencial productivo. Se expresa en kg por árbol.

Afinidad productiva $\left(A_{f}\right)$ se calcula como la relación entre las $P_{\mathrm{rFC}}$ de ambas variedades sobre un mismo portainjerto. Compara el potencial productivo de ambas variedades sobre un mismo portainjerto. Por ser adimensional mide las veces que, eventualmente, la $P_{\mathrm{rFC}}$ de una variedad supera a la de la otra.

Precocidad de producción $\left(\mathrm{P}_{\mathrm{rec}}\right)$ es un atributo productivo que mide la capacidad de un portainjerto de inducir, en una variedad, una producción temprana que supere la media (Usenik et al., 2006).

Fracción de pérdida $\left(f_{\mathrm{Per}}\right)$ es la proporción de fruta dañada o defectuosa; se expresa como g de fruta perdida por presencia de daños y defectos por cada g de fruta cosechada.

Producción fruta sana $\left(P_{r E S}\right)$ es la producción media por árbol libre de daños y de defectos macroscópicos. Es una variable calculada como el producto entre $P_{r F C}$ y el complemento de la $f_{\text {Per }}$. Representa el potencial productivo de fruta comercializable, sin la limitación de tamaño, se expresa en kg por planta.

Debido a las grandes pérdidas registradas por agrietado en el séptimo $A D I$, se estudió el efecto portainjerto e interacciones sobre $\mathrm{P}_{\mathrm{rFS}}$. La variedad Celeste y Bing experimentaron $89 \mathrm{~mm}$ y $86 \mathrm{~mm}$ de precipitación y 49 h y 54 h de mojado, respectivamente.

Volumen de dosel cono truncado $\left(\mathrm{VD}_{\mathrm{ct}}\right)$ : el cálculo asumió la forma de cono truncado, utilizando la fórmula $V D_{c t}=\pi \cdot(R 2+r 2+R r) \cdot h \cdot 1 / 3$ siendo $R=(E+L) / 2$, mientras que el radio menor $(r)$ asumió un valor de 0,40 m (Usenik et al., 2006).

\section{Análisis estadísticos}

El diseño fue por bloques completos al azar, con seis repeticiones de cuatro plantas por UE (Jiménez et al., 2007). Los datos analizados correspondieron sólo a plantas sanas. El análisis de componentes principales (ACP) y el de la varianza (ANAVA) se realizaron con el programa estadístico InfoStat (Di Rienzo et al., 2018). La prueba de comparación múltiple de medias del ANAVA fue Scott \& Knott (S\&K) y el nivel de significancia fue $p \leq 0,05$. Los coeficientes de correlación utilizados fueron de Pearson y de Spearman, según correspondió.
Los modelos de regresión fueron seleccionados con Table Curve 2D 5.0 (Systat, 2005). Se utilizó el método de comparación de regresiones anidadas (Mendenhall y Sincich, 1995).

\section{RESULTADOS Y DISCUSIÓN}

\section{Análisis exploratorio de componentes principales (ACP)}

El ACP (Figura 1) mostró que las variables edáficas que mejor representaron el efecto bloque fueron $\mathrm{CE}_{\mathrm{es}}, \% \mathrm{~L}+\mathrm{A}$ y $\mathrm{N}_{\mathrm{T}}$ las variables productivas fueron $P_{r F C}, P_{r F S}$ y $f_{\text {Per }}$ La Figura 1 representa la estructura de variación de tales variables en dos componentes principales, la primera componente (CP1) explicó el $67 \%$ de la variabilidad mientras que las dos componentes explicaron el $86 \%$ de la variabilidad total. La CP1 separó $\mathrm{CE}_{\mathrm{es}}$ y $\mathrm{f}_{\mathrm{per}}$ que explicó el comportamiento de los bloques BI y BII respecto del resto. Mientras que el comportamiento de los BIII y, especialmente, el BVI se explicó por una mayor $\mathrm{P}_{\mathrm{rFC}}$ y $\mathrm{P}_{\mathrm{rFS}}$, debido a una menor $\mathrm{CE}_{\mathrm{es}}$ y un mayor contenido de $\mathrm{N}_{\mathrm{T}}$. La CP2 separó \% $\mathrm{L}+\mathrm{A}$ que explicó el comportamiento del grupo formado por BIV y BV. El coeficiente de correlación cofenética fue 0,984

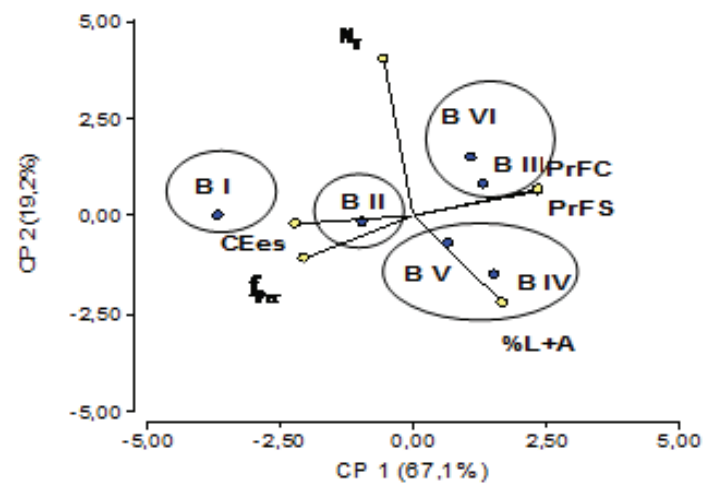

Figura 1. Gráfico biplot de análisis de componentes principales de auto-vectores clasificados según bloque

La longitud de los vectores fue semejante, lo que sugiere una contribución similar de las variables. Los autovectores $\mathrm{CE}_{\mathrm{es}}$ y $\mathrm{f}_{\mathrm{Per}}$ mostraron asociación negativa con $\mathrm{P}_{\mathrm{rFC}}$ y $\mathrm{P}_{\mathrm{rFS}}$. Mientras que $\mathrm{CE}_{\mathrm{es}}$ y $\mathrm{f}_{\mathrm{Per}}$ asociaron con magnitud positiva. El incremento de la salinidad redujo el desempeño agronómico, disminuyendo la $\mathrm{P}_{\mathrm{rFC}}$, e incrementó la $f_{\text {Per }}$, reduciendo la $P_{r F S}$. La salinidad edáfica reduce el crecimiento vegetativo y el contenido de clorofila, aumenta el estrés oxidativo y limita severamente el crecimiento de la raíz en cerezo (Erturk et al., 2007; 
López-Ortega et al., 2016; Papadakis et al., 2018).

El BI se caracterizó por presentar alta $C_{e s} y f_{\text {Per }}$ que el resto y baja $P_{r F C}$ y $P_{r F S}$. El Bll se definió por valores intermedios de $C_{e s}$ y $f_{\text {Per }}$ pero baja $P_{r F C} y$ $P_{r F S}$. Los BIII y BVI mostraron baja $C_{e s}$ y $f_{\text {Per }}$ y alta $P_{r F C}$ y $P_{r F S}$. Los BIV y BV presentaron baja CE ${ }_{e s}$ y $N_{T}$, pero valores altos de \% $L+A, P_{r F C}$ y $P_{r F S}$.

\section{Producción cosechada $\left(\mathbf{P}_{\mathrm{rFC}}\right)$}

La media de $P_{\mathrm{rFC}}$ fue 9,31 \pm 9,7 kg por árbol, con datos que oscilaron entre un mínimo de 0,0 kg y un máximo de 46,3 kg. Mostró asociación de magnitud intermedia con $V_{c t}(r=0,63 ; p \leq 0,0001)$, con una relación lineal con significancia, pero con bajo poder explicativo $\left(R^{2}=0,40 ; p \leq 0,0001\right)$ indicando que $V D_{c t}$ es un predictor impreciso de la $P_{r F C}$. Además, asoció con ADI ( $r=0,69 ; p \leq 0,0001)$, aunque la relación de causalidad tuvo un modelo lineal $\left(R^{2}=0,48 ; p \leq 0,0001\right)$ que explicó, parcialmente el comportamiento de $\mathrm{P}_{\mathrm{rFC}}$ con una tasa media anual de $4,76 \mathrm{~kg}$ por planta.

La variabilidad de $P_{r F C}$ se explicó por el modelo $\left(R_{A j}^{2}=0,82 ; p \leq 0,0001\right)$ mediante los efectos bloque $(p=0,0001), A D I(p \leq 0,0001)$, portainjerto $(p \leq 0,0001)$ e interacciones bloque-ADI ( $p \leq 0,0001)$, portainjerto-variedad ( $p \leq 0,0001)$, ADI-portainjerto ( $p \leq 0,0001)$, ADI-portainjertovariedad $(p \leq 0,0001)$ y bloque-portainjertovariedad $(p \leq 0,0001)$.

La $P_{\mathrm{rFC}}$ se inició a partir del cuarto ADI (Tabla 1), semejante a lo observado por Ivanov et al. (2018) y desde el cuarto hasta el octavo ADI se incrementó sistemáticamente por lo que no se alcanzó una meseta productiva (Tabla 1). El mayor incremento de $P_{r F C}$ fue al sexto ADI, con $8,6 \mathrm{~kg}$ respecto del quinto.

La Figura 2 muestra el comportamiento general del ensayo, a través de la interacción bloque-ADI. El BI no superó la media, durante los cinco ADI mientras que, a partir del BII, lo hicieron desde el sexto $A D I$, indicando que la menor fertilidad debida a una mayor $\mathrm{CE}_{\mathrm{es}}$, reduce la $\mathrm{P}_{\mathrm{rec}}$. El efecto variedad no tuvo significancia $(p \leq 0,6380)$.

El mayor potencial productivo representado por $P_{r F C}$ se registró sobre los portainjertos SL 64, MxM 60 y Adara-M2624. Godini et al. (2008) observaron mayor rendimiento sobre SL 64 que sobre MxM 60 , indicando que, en bajas latitudes con veranos cálidos, el mejor comportamiento correspondió a clones de P. mahaleb. Mientras que López Ortega et al. (2016) reportaron mayor producción sobre Adara-M2624 que sobre SL 64. Además, Jiménez et al. (2007) encontraron que Adara, utilizado como

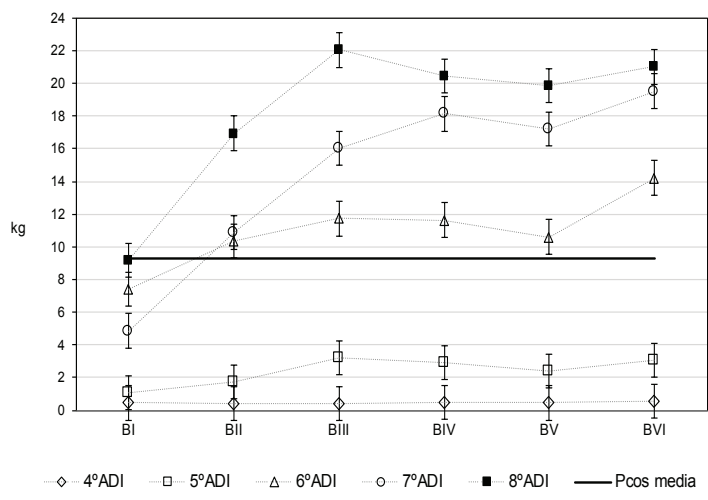

Figura 2. Efecto interacción bloque-ADI sobre la producción cosechada por árbol. Test de Scott \& Knott $(\alpha=0,05)$

portainjerto, alcanzó la mayor producción entre los vigorosos. Le siguió el grupo formado por W 13 y MxM 14 con valores intermedios. Los trabajos de Cantín et al. (2010), Fajt, Folini et al. (2014) y LópezOrtega et al. (2016) reportaron menor producción sobre MxM 14 que sobre Adara, aunque semejante a la de SL 64. Luego, un tercer grupo formado por CAB $6 P$ y $G$ con valores menores que la media. Gratacós et al. (2007) encontraron que Bing sobre CAB $6 \mathrm{P}$ fue menos productivo que sobre MxM 14, pero Jiménez et al. (2007) reportaron que CAB 6P no diferenció de MxM 14 o SL 64. La menor $P_{\mathrm{rFC}}$ fue sobre G 5, consistente a lo observado por Gratacós et al. (2007), Jiménez et al. (2007) y López Ortega et al. (2016) (Tabla 1).

La interacción portainjerto-variedad (Tabla 1) mostró que la mayor $\mathrm{P}_{\mathrm{rFC}}$ fue alcanzada por Celeste sobre SL 64 o Adara-M2624 con incrementos sobre la media del $56 \%$ y del $74 \%$, respectivamente. Consistente a lo señalado por Long y Kaiser (2010) y Sansavini y Lugli (2014), que indicaron que las variedades autofértiles son más productivas sobre portainjertos de alto vigor. Le siguieron Celeste sobre MxM 60 y Bing sobre SL 64, MxM 60 o W 13 con incrementos sobre la media del $33 \%$ y del $13 \%, 36 \%$ y $22 \%$, respectivamente. Luego, un tercer grupo, con valores intermedios, compuesto por Bing sobre MxM 14, CAB 6P, G 6 o Adara-M2624 y Celeste sobre MxM 14, CAB 6P y W 13. La variedad Bing sobre $G 5$ y Celeste sobre $\mathrm{G} 6$ redujeron la $\mathrm{P}_{\mathrm{rFC}}$ al $50 \%$ de la media. La menor $P_{\mathrm{rFC}}$ la registró Celeste sobre G 5 con el $24 \%$ de la media.

La Figura 3 muestra que la variedad Celeste sobre los portainjertos SL 64 o Adara-M2624 registró 1,38 y 1,85 veces mayor $A_{f}$ que Bing sobre los mismos portainjertos, respectivamente (Tabla 1). La evidencia indica que variedades autofértiles de vigor medio inducen abundante carga frutal 
Tabla 1. Efecto bloque, ADI, portainjerto, variedad e interacción portainjerto-variedad sobre la $P_{r F C}, P_{r F S} f_{P e r} y V D_{c t}$

\begin{tabular}{|c|c|c|c|c|c|}
\hline \multicolumn{2}{|c|}{ Efectos } & \multirow{2}{*}{$\frac{{ }^{2} \mathrm{P}_{\mathrm{rFC}}(\mathrm{kg} / \mathrm{pta})}{4,6 \mathrm{a}}$} & \multirow{2}{*}{$\frac{{ }^{3} \mathrm{P}_{\mathrm{rFS}}(\mathrm{kg} / \mathrm{pta})}{2,8 \mathrm{a}}$} & \multirow{2}{*}{$\frac{{ }^{4} f_{\text {Per }} g^{-1}}{0.39}$} & \multirow{2}{*}{$\frac{{ }^{5} \mathrm{VD}_{\mathrm{ct}}\left(\mathrm{m}^{3}\right)}{9,9 \mathrm{a}}$} \\
\hline \multirow{6}{*}{ Bloque } & I & & & & \\
\hline & II & $8,1 \mathrm{~b}$ & $4,9 \mathrm{~b}$ & 0,40 & $15,2 b$ \\
\hline & III & $10,7 \mathrm{c}$ & $6,9 \mathrm{c}$ & 0,36 & $19,6 \mathrm{c}$ \\
\hline & IV & $10,7 \mathrm{c}$ & $6,8 \mathrm{c}$ & 0,35 & $19,0 \mathrm{c}$ \\
\hline & V & $10,1 \mathrm{c}$ & $6,0 \mathrm{c}$ & 0,36 & $18,2 \mathrm{c}$ \\
\hline & $\mathrm{VI}$ & $11,7 \mathrm{c}$ & $7,2 \mathrm{c}$ & 0,34 & $19,0 \mathrm{c}$ \\
\hline \multirow{5}{*}{${ }^{1} \mathrm{ADI}$} & 4 to & $0,5 \mathrm{a}$ & $0,4 \mathrm{a}$ & $0,23 \mathrm{a}$ & $12,2 \mathrm{a}$ \\
\hline & 5 to & $2,4 b$ & $1,5 b$ & $0,38 \mathrm{~b}$ & $14,6 \mathrm{~b}$ \\
\hline & 6 to & $11,0 \mathrm{c}$ & $7,4 \mathrm{~d}$ & $0,35 \mathrm{~b}$ & $19,4 d$ \\
\hline & 7 to & $14,4 d$ & $5,9 \mathrm{c}$ & $0,61 \mathrm{c}$ & $19,7 d$ \\
\hline & $8 \mathrm{vo}$ & $18,2 \mathrm{e}$ & $13,6 \mathrm{e}$ & $0,26 \mathrm{a}$ & $18,1 \mathrm{c}$ \\
\hline \multirow{8}{*}{ Portainjerto } & SL 64 & $12,5 d$ & $7,9 \mathrm{c}$ & 0,35 & $24,1 \mathrm{~g}$ \\
\hline & MxM 60 & $12,5 d$ & $8,0 \mathrm{c}$ & 0,35 & $22,9 f$ \\
\hline & MxM 14 & $9,1 \mathrm{c}$ & $5,5 b$ & 0,39 & $18,5 d$ \\
\hline & CAB 6P & $7,4 \mathrm{~b}$ & $4,5 b$ & 0,37 & 20,3 e \\
\hline & W1 3 & $10,1 \mathrm{c}$ & $6,6 \mathrm{c}$ & 0,36 & $15,8 \mathrm{c}$ \\
\hline & G 5 & $3,4 \mathrm{a}$ & $2,2 \mathrm{a}$ & 0,37 & $4,7 \mathrm{a}$ \\
\hline & G 6 & $6,8 \mathrm{~b}$ & $4,1 \mathrm{~b}$ & 0,37 & 8,8 b \\
\hline & Adara & $12,5 d$ & $7,3 \mathrm{c}$ & 0,37 & $19,2 d$ \\
\hline \multirow{2}{*}{ Variedad } & Bing & 9,3 & $6,5 b$ & $0,27 \mathrm{a}$ & $19,2 \mathrm{~b}$ \\
\hline & Celeste & 9,3 & $5,0 \mathrm{a}$ & $0,46 \mathrm{~b}$ & $14,4 \mathrm{a}$ \\
\hline \multirow{16}{*}{$\begin{array}{l}\text { Portainjerto- } \\
\text { variedad }\end{array}$} & B-SL 64 & $10,5 d$ & $7,5 \mathrm{e}$ & $0,27 \mathrm{a}$ & $25,3 \mathrm{i}$ \\
\hline & B-MxM 60 & $12,7 d$ & $8,5 \mathrm{e}$ & $0,27 \mathrm{a}$ & 25,3 i \\
\hline & B-MxM1 4 & $9,8 \mathrm{c}$ & $6,4 \mathrm{~d}$ & $0,34 a$ & $20,5 \mathrm{~g}$ \\
\hline & B-CAB 6P & $7,7 \mathrm{c}$ & $5,3 \mathrm{c}$ & $0,29 a$ & $21,7 \mathrm{~g}$ \\
\hline & B-W 13 & $11,4 d$ & $8,0 \mathrm{e}$ & $0,28 \mathrm{a}$ & $18,5 f$ \\
\hline & B-G 5 & 4,6 b & $3,5 b$ & $0,22 \mathrm{a}$ & $7,3 \mathrm{c}$ \\
\hline & B-G 6 & $9,0 \mathrm{c}$ & $6,2 \mathrm{~d}$ & $0,27 \mathrm{a}$ & $12,1 d$ \\
\hline & B-Adara & $8,8 \mathrm{c}$ & $6,6 \mathrm{~d}$ & $0,25 a$ & $23,0 \mathrm{~h}$ \\
\hline & C-SL 64 & $14,5 \mathrm{e}$ & $8,4 \mathrm{e}$ & $0,44 \mathrm{~b}$ & $22,9 \mathrm{~h}$ \\
\hline & C-MxM 60 & $12,3 d$ & $7,4 \mathrm{e}$ & $0,42 \mathrm{~b}$ & $20,6 \mathrm{~g}$ \\
\hline & C-MxM 14 & $8,4 \mathrm{c}$ & $4,6 \mathrm{c}$ & $0,43 \mathrm{~b}$ & $16,5 \mathrm{e}$ \\
\hline & C-CAB 6P & $7,2 \mathrm{c}$ & $3,7 \mathrm{~b}$ & $0,46 b$ & $18,9 f$ \\
\hline & C-W 13 & $8,9 \mathrm{c}$ & $5,1 \mathrm{c}$ & $0,43 \mathrm{~b}$ & $13,1 d$ \\
\hline & C-G 5 & $2,3 a$ & $1,0 \mathrm{a}$ & $0,51 \mathrm{c}$ & $2,2 \mathrm{a}$ \\
\hline & C-G 6 & 4,6 b & $2,2 \mathrm{a}$ & $0,47 \mathrm{c}$ & $5,5 \mathrm{~b}$ \\
\hline & C-Adara & $16,2 \mathrm{e}$ & $7,9 \mathrm{e}$ & $0,50 \mathrm{c}$ & 15,3 e \\
\hline
\end{tabular}

Para cada columna y efecto, las medias seguidas por una letra común no son significativamente diferentes según el Test de Scott \& Knott $(p>0,05) .{ }^{1} \mathrm{ADI}$ (Años Después de la injertación), ${ }^{2} \mathrm{P}_{\mathrm{rFC}}$ (Producción media cosechada por árbol), ${ }^{3} \mathrm{P}_{\mathrm{rFS}}$ (Producción media de fruta sana por árbol), ${ }^{4 f_{\text {Per }}}$ (Fracción de pérdida), ${ }^{7} \mathrm{VD}_{\mathrm{ct}}$ (Volumen de dosel cono truncado).

sobre portainjertos vigorosos o semi-vigorosos (Robinson et al., 2008; Long y Kaiser, 2010; Sansavini y Lugli, 2014). La variedad Bing sobre los portainjertos G 5, G 6 o W 13 registró 2,05; 1,96 y 1,29 veces mayor $A_{f}$ que Celeste sobre los mismos portainjertos, respectivamente. Según, Sansavini y Lugli (2014) los portainjertos enanizantes mejoran el desempeño productivo de variedades autoincompatibles. Así mismo, Long y Kaiser (2010) indicaron que los portainjertos semienanizantes son los indicados para cultivar variedades de baja a media productividad. Por lo tanto, para alcanzar una alta $P_{r F C}$ se deben seleccionar combinaciones específicas de portainjerto-variedad. Los trabajos de Robinson et al. (2008) y de Sansavini y Lugli (2014) mencionan que los portainjertos mejoran el desempeño productivo de una variedad en relación a otra.

Por otro lado, existen portainjertos como MxM 60, MxM 14 o CAB 6P que no diferencian $\mathrm{P}_{\mathrm{rFC}}$ entre variedades y consecuentemente no presentan $A_{f}$, considerándose portainjertos estables al cambio 


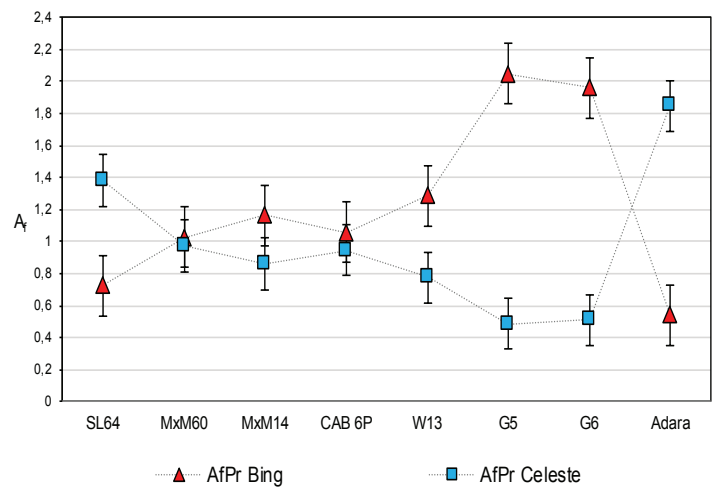

Figura 3. Efecto interacción portainjerto-variedad sobre la afinidad productiva. Test de Scott \& Knott $(\alpha=0,05)$

varietal, aunque alcanzan sólo valores de $\mathrm{P}_{\mathrm{rFC}}$ intermedios (Tabla 1).

La Figura 4 muestra el efecto interacción ADI-portainjerto sobre la $P_{\mathrm{rFC}}$. Los árboles sobre SL 64, MxM 60 o Adara-M2624 superaron consistentemente la $\mathrm{P}_{\mathrm{rFC}}$ media, a partir del sexto ADI. Sin embargo, la interacción ADI-portainjertovariedad de la Figura 5 muestra que sólo las combinaciones de Bing sobre SL 64, MxM 60, W 13 o G 6 y las de Celeste sobre SL 64, MxM 60 o Adara-M2624, superaron la $P_{\mathrm{rFC}}$ media, a partir del sexto $A D I$, siendo las de mayor $P_{\text {rec }}$. Las combinaciones de Bing con G 5 o de Celeste con G 5 o $G 6$ mostraron baja $P_{\mathrm{rFC}}$ (datos no mostrados) probablemente, debido a la falta de adaptación a condiciones de clima árido (Fajt, Jug et al., 2014; Sansavini y Lugli, 2014).

La Figura 6 muestra que Bing sobre MxM 60 o W 13 superaron la $P_{r F C}$ en cinco de los seis bloques y que MxM 14 o G 6 lo hicieron en la mitad de los bloques. La variedad Celeste superó la media sobre SL 64 o Adara-M2624 en cinco de los seis bloques y MxM 60 lo hizo en cuatro bloques.

Se destaca el desempeño de Bing sobre el semienanizante W 13 o sobre el enanizante G 6 , con doseles de tamaño reducido (Tersoglio y Setién, 2016), que posibilitan el aumento de la densidad de plantación y eventual, incremento del rendimiento, consistente con lo mencionado por Long y Kaiser (2010).

\section{Producción de fruta sana $\left(\mathrm{P}_{\mathrm{RFS}}\right)$}

La $P_{\mathrm{rFS}}$ media fue $5,76 \pm 6,8 \mathrm{~kg}$ por planta, que correspondió al $62 \%$ de la $P_{r F C}$, con valores que oscilaron entre un mínimo de $0,0 \mathrm{~kg}$ y un máximo de $32,2 \mathrm{~kg}$.

La $P_{r F S}$ asoció con $V D_{\text {ct }}(r=0,56 ; p \leq 0,0001)$, con

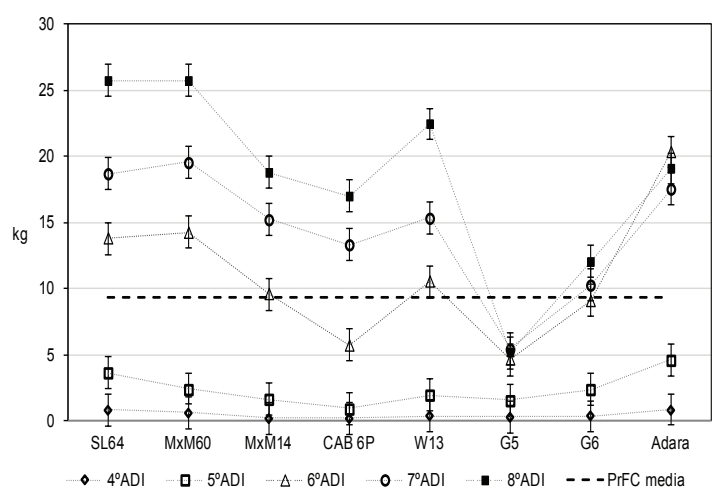

Figura 4. Efecto interacción ADI-portainjerto sobre la producción cosechada. Test de Scott \& Knott $(\alpha=0,05)$

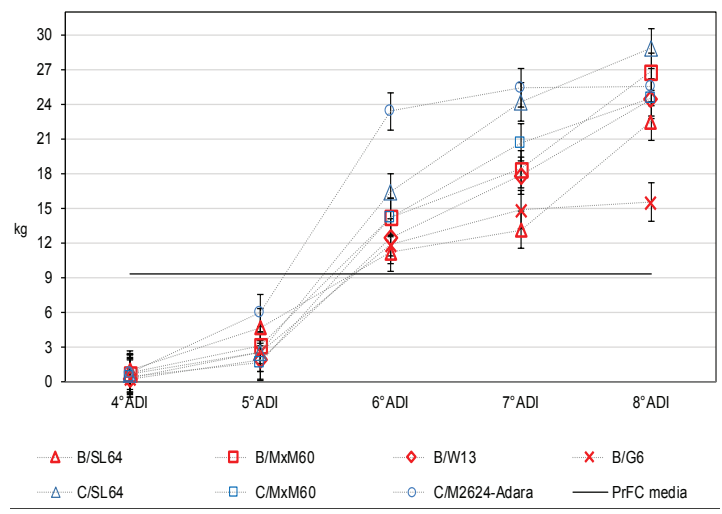

Figura 5. Combinaciones portainjerto-variedad que superaron la media de producción cosechada con mayor frecuencia. Test de Scott \& Knott $(\alpha=0,05)$

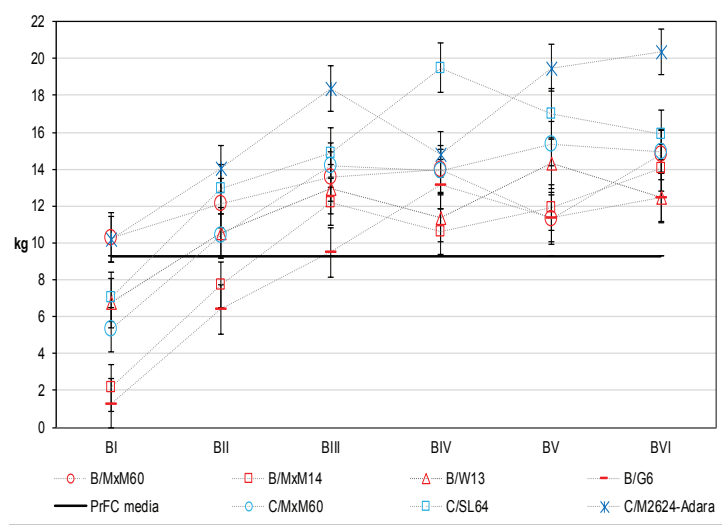

Figura 6. Combinaciones portainjerto-variedad que superaron la media de producción cosechada, en mayor número de bloques. Test de Scott \& Knott $(\alpha=0,05)$

una relación $\left(R^{2}=0,31 ; p \leq 0,0001\right)$ y también con ADI $(r=0,74 ; p \leq 0,0001)$ con una relación $\left(R^{2}=0,41\right.$; $p \leq 0,0001)$. Ambos modelos fueron lineales, con significancia, pero de bajo poder explicativo. Asoció con variedad $(r=0,45 ; p \leq 0,0001)$ y lo hizo 
con alta magnitud con $\mathrm{P}_{\mathrm{rFC}}(r=0,90 ; p \leq 0,0001)$, con una relación lineal $\left(R^{2}=0,85 ; p \leq 0,0001\right)$, indicando que la $P_{r F S}$ fue altamente dependiente de $P_{\mathrm{rFC}}$ en las condiciones del ensayo.

La variabilidad de $P_{r F S}$ fue explicada por el modelo $\left(R_{A j}^{2}=0,79 ; p \leq 0,0001\right)$ a través de los efectos bloque $(p \leq 0,0001), A D I \quad(p \leq 0,0001)$, portainjerto $(p \leq 0,0001)$, variedad $(p \leq 0,0001)$, interacción portainjerto-variedad $(p \leq 0,0001)$, ADI-portainjerto-variedad $(p=0,0039)$ y bloqueportainjerto-variedad $(p=0,0092)$.

La Tabla 1 muestra que $P_{r F S}$, al igual que $P_{r F C}$ se inició al cuarto ADI con el $7 \%$ de la media, hasta un incremento del $136 \%$ sobre la media, en el octavo ADI. Pero, a diferencia de la $P_{r F C}$, la $P_{r F S}$ del séptimo $A D I$ fue inferior a la del sexto $A D I$, debido a la alta $f_{\text {Per, }}$ consecuencia de condiciones climáticas adversas durante la cosecha (Tabla 1).

La $P_{r F S}$ de Bing fue $29 \%$ mayor que la de Celeste (Tabla 1), indicando la importancia de la selección varietal.

Los portainjertos SL 64, MxM 60, W 13 o Adara-M2624 registraron la mayor $P_{r F S}$, mientras que, la de $\mathrm{G} 5$ registró la menor (Tabla 1).

La mayor $\mathrm{P}_{\mathrm{rFS}}$ de la variedad Bing se registró sobre SL 64, MxM 60 o W 13 seguidos por MxM14, G 6 o Adara-M2624 con incrementos del 30\%, $47 \%$ y $39 \%$ y del $11 \%, 7 \%$ y $15 \%$ sobre la media, respectivamente. Mientras que la variedad Celeste alcanzó la mayor $\mathrm{P}_{\mathrm{rFS}}$ sólo sobre SL 64, MxM 60 o Adara-M2624 con incrementos del 46\%, 29\% y $37 \%$ sobre la media, respectivamente. El resto de las combinaciones tuvo $\mathrm{P}_{\mathrm{rFS}}$ menor que la media. La variedad Bing sobre CAB 6P y Celeste sobre MxM 14 o W 13 redujeron la $P_{r F S}$ al $91 \%, 79 \%$ y $89 \%$ de la media, respectivamente. El grupo formado por Bing sobre G 5 y Celeste sobre CAB $6 P$ la redujo al $61 \%$ y al $64 \%$ de la media, respectivamente. La menor $P_{r F S}$ correspondió a Celeste sobre $G$ 5 o G 6 con reducciones al $16 \%$ y al $38 \%$ de la media, respectivamente (Tabla 1). Se destaca que, excepto el muy enanizante G 5, la variedad Bing registró alta $P_{r F S}$ sobre portainjertos de diferente vigor, mientras que, Celeste lo hizo sólo sobre portainjertos vigorosos, resaltando la importancia de la correcta selección de la combinación (Aglar y Yıldız, 2014; Fajt, Jug et al., 2014; Sansavini y Lugli, 2014; Gainza et al., 2015).

Se resalta el buen desempeño de Bing sobre W 13 que es semienanizante y $G 6$ que es enanizante, lo cual posibilita incrementar la densidad de plantación.

La Figura 7 muestra que las combinaciones de variedad-portainjerto Bing sobre MxM 60, W 13 o

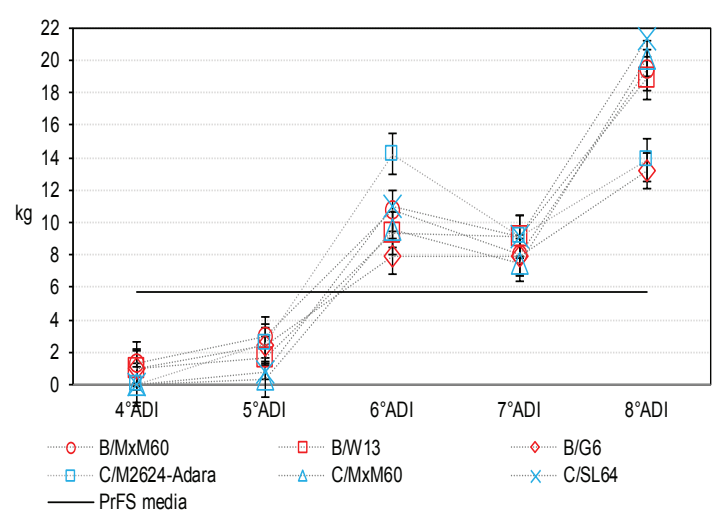

Figura 7. Combinaciones portainjerto-variedad que superaron la media de la producción de fruta sana, con mayor frecuencia. Test de Scott \& Knott $(\alpha=0,05)$

G 6 y Celeste sobre SL 64, MxM 60 o Adara-M2624 superaron la $P_{\mathrm{rFS}}$ media más frecuentemente, en tres de los cinco ADI.

La Figura 8 muestra las combinaciones variedad-portainjerto que superaron la $P_{\mathrm{rFS}}$ media en el mayor número de bloques. La variedad Bing lo hizo sobre MxM 60, seguida por W 13 , en cinco y cuatro de los seis bloques, respectivamente y luego, MxM 14, G 6 o Adara-M2624 que lo hicieron en la mitad de los bloques. La variedad Celeste lo hizo sobre SL 64, en cinco de los seis bloques y sobre MxM 60 o Adara-M2624 lo hicieron en cuatro de los seis bloques.

Debido a que el séptimo ADI registró la mayor $f_{\text {Per }}$ (Tabla 1), se analizó en particular, la influencia del efecto interacción portainjerto-variedad sobre la $P_{r F S}$. La variabilidad de $P_{r F S}$ del séptimo ADI fue explicada por el modelo $\left(R^{2}=0,79 ; p=0,0062\right)$ a través de los efectos bloque $(p=0,0004)$, portainjerto $(p=0,0196)$, variedad $(p=0,0093)$ e interacción portainjerto-variedad $(p=0,0013)$. La Figura 9 muestra que la media de $P_{r F S}$ de la variedad Bing registró $6,9 \mathrm{~kg}$ y la de Celeste midió $4,9 \mathrm{~kg}$. La variedad Bing superó la media varietal sobre MxM 60, MxM 14 o W 13 y Celeste lo hizo sobre SL 64, MxM 60 o Adara-M2624 con incrementos sobre la correspondiente media varietal del 20, 42 y $37 \%$, y del 81,47 y $81 \%$, respectivamente.

\section{Fracción de pérdida $\left(f_{\mathrm{Per}}\right)$}

La $f_{\text {Per }}$ media fue $0,37 \pm 0,22 \mathrm{~g}$ de fruta dañada o defectuosa por cada $g$ de fruta cosechada. La $f_{\text {Per }}$ sólo asoció con variedad $(r=0,45 ; p \leq 0,0001)$. El modelo $\left(R_{A j}^{2}=0,79 ; p \leq 0,0001\right)$ explicó la variabilidad mediante los efectos ADI ( $p \leq 0,0001)$, variedad $(p \leq 0,0001)$ e interacciones portainjerto- 


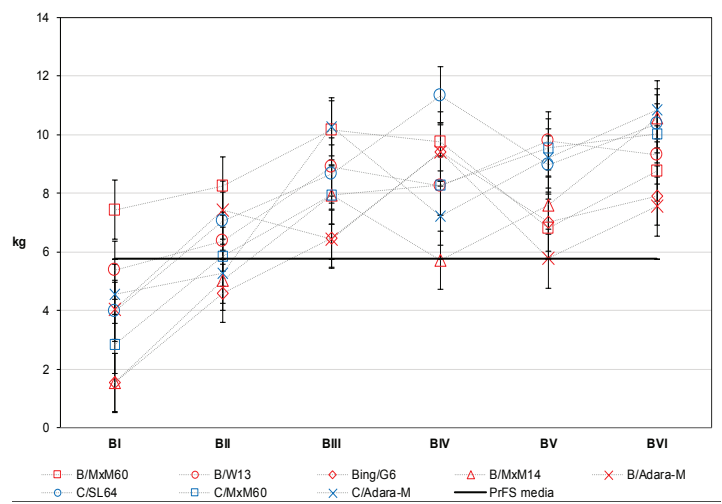

Figura 8. Combinaciones portainjerto-variedad que superan la media de la producción de fruta sana, en mayor número de bloques. Test de Scott \& Knott $(\alpha=0,05)$

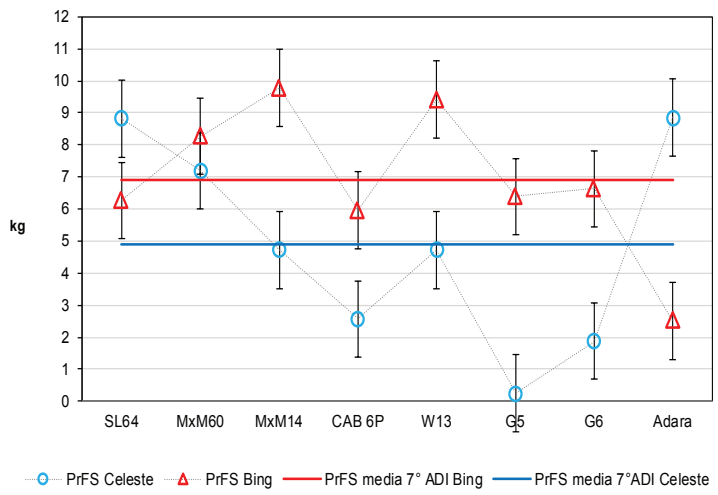

Figura 9. Efecto interacción portainjerto-variedad del $7^{\circ} \mathrm{ADI}$, sobre producción de fruta sana. Test de Scott \& Knott $(\alpha=0,05)$

variedad $(p=0,0008)$, bloque-portainjerto-variedad $(p=0,002)$ y ADI-portainjerto-variedad $(p=0,0027)$.

La menor $\mathrm{f}_{\text {per }}$ se registró al cuarto y al octavo ADI y la mayor al séptimo ADI con un incremento de $0,24 \mathrm{~g} \cdot \mathrm{g}^{-1}$ sobre la media (Tabla 1). La variedad Celeste registró un aumento de la $\mathrm{f}_{\mathrm{Per}}$ de $0,19 \mathrm{~g} \cdot \mathrm{g}^{-1}$, que correspondió a un incremento del $70 \%$ respecto de la de Bing (Tabla 1). La variedad Celeste midió, consistentemente, mayor $f_{\text {Per }}$ durante los cinco ADI y en todos los bloques (datos no mostrados). Portainjerto no tuvo significancia $(p=0,6308)$, al igual que bloque $(p=0,1283)$.

La interacción portainjerto-variedad (Tabla 1) muestra que Bing registró menor $\mathrm{f}_{\mathrm{per}}$ que Celeste sobre los portainjertos estudiados, indicando la gran influencia de la variedad. Mientras que, Celeste sobre G 5, G 6 o Adara-M2624 registró $f_{\text {Per }}$ con incrementos del $38 \%, 27 \%$ y $35 \%$ sobre la media. El resto de los portainjertos registraron aumentos menores que oscilaron entre $14 \%$ y el $24 \%$ sobre la media (Tabla 1 ).

\section{CONCLUSIONES}

Unamayor $\mathrm{CE}_{\mathrm{es}}$ reduce laproducción cosechada, la de fruta sana y la precocidad. En las condiciones del ensayo, $P_{r E S}$ fue adecuadamente explicada por la $P_{r F C}$. La afinidad productiva entre variedad y portainjerto tiene alta especificidad en cerezos. La información indica que las variedades mejoran la producción sobre algunos portainjertos vigorosos pero, las auto-incompatibles lo hacen, además, sobre los semienanizantes mientras que, las autocompatibles lo hacen sobre los semivigorosos. La $P_{r F C}$, la $P_{r F S}$ y la $f_{\text {Per }}$ fue afectada por portainjerto $y$ sus interacciones con variedad, ADI y bloque, indicando que la selección de una combinación debe considerar, además de la producción, la adaptación edafo-climática a las condiciones locales. La mayor producción cosechada se logró sobre SL 64, MxM 60, W 13 y Adara-M2624. Las combinaciones que además, registraron el mayor potencial productivo, precocidad y adaptación a las condiciones edafo-climáticas fueron Bing sobre MxM 60 o W 13 y Celeste sobre SL 64, MxM 60 o Adara-M2624. La mayor producción de fruta sana se logró sobre SL 64, MxM 60, W 13 o Adara-M2624. Las combinaciones que registraron mayor adaptación a las condiciones edafoclimáticas y mejor respuesta bajo condiciones de Iluvia fueron Bing sobre MxM 60 o W 13 y Celeste sobre SL 64, MxM 60 o Adara-M2624.

\section{AGRADECIMIENTO}

Este trabajo fue posible gracias a la valiosa colaboración de los viveros de Frutales Puertas Hnos., Agroviveros SA y Bobafrut SRL.

\section{BIBLIOGRAFÍA}

Aglar, E. y Yıldız, K. (2014). Influence of rootstocks (Gisela 5, Gisela 6, MaxMa, SL 64) on performance of '0900 Ziraat' sweet cherry. Journal of Basic and Applied Sciences, 10, 60-66.

Ampatzidis, Y. y Whiting, M. (2013). Training system affects sweet cherry harvest efficiency. HortScience, 48(5), 547-555.

Cantín, C., Pinochet, J., Gorgoncena, Y. y Moreno, M. (2010). Growth, yield and fruit quality of 'Van' and 'Stark Hardy Giant' sweet cherry cultivars as influenced by grafting on different rootstocks. Scientia Horticulturae, 123(3), 329-335.

Erturk, U., Sivritepe, N., Yerlikaya, C., Bor, M., Ozdemir, F. y Turkan, I. (2007). Responses of the cherry rootstock to salinity in vitro. Biologia Plantarum, 51(3), 597-600. 
Fajt, N., Jug, T., Biško, A., Purgaj, B., Folini, L., Stainbauer, L. y Usenik, V. s.f. AlpeAdria Trial - Sweet cherry Regina on three dwarf rootstocks and different plant distances. Recuperado de:

https://www.bordeaux.inra.fr/cherry/docs/dossiers/ Activities/Meetings/2014\%2010\%2013-15 4th\%20 MC\%20and\%20all\%20WG\%20Meeting Bordeaux/ Posters/Fajt_Bordeaux2014.pdf

Fajt, N., Folini, L., Bassi, G. y Siegler, H. (2014). Lapins' on ten cherry rootstocks in the Alpe Adria Region. Acta Horticulturae, 1020, 371-376.

Gainza, F., Opazo, I., Guajardo, V., Meza, P., Ortiz, M., Pinochet, J. y Muñoz, C. (2015). Rootstock breeding in Prunus species: Ongoing efforts and new challenges.Chilean Journal of Agricultural Research, 75, 6-16. https://dx.doi.org/10.4067/S071858392015000300002

Godini, A., Palasciano, M., Ferrara, G., Camposeo, S. y Pacifico, A. (2008). On the advancement of bud break and fruit ripening induced by hydrogen cyanamide (Dormex®) in sweet cherry: A three-year study. Acta Horticulturae, 795, 469-477.

Gonçalves, B., Moutinho-Pereira, J., Santos, A., Silva, A., Bacelar, E., Correia, C. y Rosa, E. (2006). Scionrootstock interaction affects the physiology and fruit quality of sweet cherry. Tree Physiology, 26, 93-104.

Gratacós, N., Cortés, B. y Kulczewski, B. (2007). New rootstocks for the Chilean cherry industry. Compact Fruit Tree, 4, 19-21.

Ivanov, I., Balan, V., Pe teanu, A., Vamasescu, S., Balan, P. y Sarban, V. (2018). Influence of the planting distance and the crown shape on the fruit harvest and the productive potential of cherry trees in a high-density system. Bulletin UASVM Horticulture, 75(2), 163-168. dx.doi.org/10.15835/buasvmcn-hort:2018.0014

Jiménez, S., Pinochet, J., Gogorcena, Y., Betrán, J. y Moreno, M. (2007). Influence of different vigour cherry rootstocks on leaves and shoots mineral composition. Scientia Horticulturae, 112(1), 73-79.

Ljubojević, M., Ognjanov, V., Barać, G., Dulić, J., Miodragović, M., Sekulić, M. y Jovanović, N. (2016). Cherry tree growth models for orchard management improvement. Turkish Journal of Agriculture and Forestry, 40, 839-854. https://doi.org/10.3906/tar1606-14

Long, L. y Kaiser, C. (2010). Sweet cherry roostocks for the Pacific Northwest [Documento PDF]. Pacific Northwest Extension Publishing. https://catalog. extension.oregonstate.edu/sites/catalog/files/project/ pdf/pnw619.pdf

López-Ortega, G., García-Montiel, F., Bayo-Canhaa, A., Frutos-Ruiza, C. y Frutos-Tomás, D. (2016). Rootstock effects on the growth, yield and fruit quality of sweetcherry cv. 'Newstar' in the growing conditions of the Region of Murcia. Scientia Horticulturae, 198, 326335. https://doi.org/10.1016/j.scienta.2015.11.041

Measham, P., Gracie, A., Wilson, S. y Bound, A. (2010). Vascular flow of water induces side cracking in sweet cherry (Prunus avium L.). Advances in Horticultural Science, 24(4), 243-248.

Measham, P., Wilson, S., Gracie, A. y Bound, S. (2014). Tree water relations: flow and fruit. Agriculture Water Management, 137, 59-67.

Mendenhall, W. y Sincich, T. (1995). Statistics for engineering and the sciences (4a. edición). Prentice Hall.

Moreno, M., Tabuenca, M. y Cambra, R. (1995).Adara, a plum rootstock for cherries and other stone fruit species. Hortscience, 30(6), 1316-1317.

Papadakis, I., Veneti, G., Chatzissavvidis, C. y Therios, I. (2018). Physiological and growth responses of sour cherry (Prunus cerasus L.) plants subjected to shortterm salinity stress. Acta Botanica Croatica, 77(2), 197-202.

Pimentel, P., Almada, R., Salvatierra, A., Toro, G., Arismendi, M., Pino, M.T., Sagredo, B. y Pinto, M. (2014). Physiological and morphological responses of Prunus species with different degree of tolerance to long-term root hypoxia. Scientia Horticulturae, 180, 1423. https://doi.org/10.1016/j.scienta.2014.09.055

Rana, R., Andriano, A., Giungato, P. y Tricase, C. (2019). Carbon footprint of processed sweet cherries (Prunusavium L.): From nursery to market. Journal of Cleaner Production, 227, 900-910. https://doi. org/10.1016/j.jclepro.2019.04.162

Robinson, T., Andersen, R. y Hoying, S. (2008). Performance of Gisela $₫$ rootstocks in six high density sweet cherry training systems in the northeastern United States. Acta Horticulturae, 795, part 1, 245254.

Sansavini, S. y Lugli, S. (2014). New rootstocks for intensive sweet cherry plantations. Acta Horticulturae, 1020, 411-434.

Schumann C. y Knoche M. (2020). Swelling of cell walls in mature sweet cherry fruit: factors and mechanisms. Planta, 251,_65. https://doi.org/10.1007/s00425-02003352-y

Seavert, C. F. y Long, L. E. (2007). Financial and economic comparison between establishing a standard and high density sweet cherry orchard in Oregon, USA. Acta Horticulturae, 732, 501-504.

Serradilla, M., Hernández, A., López-Corrales, M., RuizMoyano, S., Guía-Córdoba, M. y Martín, A. (2016). Composition of the cherry (Prunus avium L. and Prunus cerasus L.; Rosaceae). En: M. Simmonds y V. R. Preedy (Eds), Nutritional composition of fruit 
cultivars (127-147). https://doi.org/10.1016/B978-012-408117-8.00006-4

Silva, H. y Rodriguez, J. (1995). Eficiencia y cálculo de la dosis de la fertilización nitrogenada. En: C. Bonomelli, (Ed.), Fertilización de plantaciones frutales (177-212). Pontificia Universidad Católica de Chile

Systat. Tablecurve-2D $\AA$ (2005, version de prueba) [Software]. https://web.archive.org/ web/20070427231959/http://www.systat.com/ downloads/?sec $=\mathrm{d} 002$

Tersoglio, E. y Naranjo, G. (2007). Características del frío invernal de las zonas productoras de cerezas de la provincia de Mendoza, Argentina. Parte I. Información Técnica Económica Agraria, 103(4), 186-197.
Tersoglio, E. y Setien, N. (2016). Efecto de la combinación Adara-M2624 y otros siete portainjertos sobre las características del dosel de dos variedades de cerezo. Agriscientia, 33(2), 113-125.

Usenik, V., Fajt, N. y Štampar, F. (2006). Effects of rootstocks and training system on growth, precocity and productivity of sweet cherry. Journal of Horticultural Science and Biotechnology, 81(1), 153-157.

Whiting, M., Lang, G. y Ophardt, D. (2005). Rootstocks and training system affect sweet cherry growth, yield and fruit quality. HortScience, 40(3), 582-586.

Winkler, A., Brüggenwirth, M., Ngo, N. y Knoche, M. (2016). Fruit apoplast tension draws xylem water into mature sweet cherries. Scientia Horticulturae, 209, 270-278. 